\title{
An Unusual Case of Bacterial Meningitis
}

\author{
(1) Mian Jie Lim, (1) Jen Heng Pek
}

Department of Emergency Medicine, Sengkang General Hospital, SingHealth, Singapore

\begin{abstract}
Bacterial meningitis is a medical emergency that requires prompt identification and treatment in the emergency department (ED). Medical management is the mainstay of treatment. However, some patients may require neurosurgical intervention. Furthermore, neurological complications may develop, causing significant morbidity and mortality. We present a case report of a 42-year-old Chinese gentleman who presented to the ED and was subsequently diagnosed with pneumococcal meningitis. In addition to routine medical management, he also underwent emergent extraventricular drainage procedure to alleviate increased intracranial pressure. He subsequently developed stroke, seizure and sensorineural hearing loss. Not seizure-related hearing loss. After a comprehensive rehabilitation program, he showed good response and recovered without focal neurological deficit. A high index of suspicion and timely interventions in ED are important in minimizing morbidity and mortality from bacterial meningitis. The help of neurosurgeons may be necessary in selected cases. Dexamethasone has an important role in the prevention of neurological complications, as well as in their treatment.
\end{abstract}

Keywords: Emergency department, meningitis, neurosurgical procedure

\section{Introduction}

Bacterial meningitis (BM) is an inflammation of the leptomeninges and tissues surrounding the brain and spinal cord due to a bacterial infection - most commonly due to Streptococcus pneumonia and Neisseria meningitidis, which results in an elevated number of white blood cells in cerebrospinal fluid (CSF) (1). The incidence of BM is estimated to be around 0.6 to 4 per 100,000 adults per year, with higher incidence in developing countries (2). Fortunately, the availability of vaccines for BM has resulted in a significant reduction in overall incidence.

Nonetheless, BM still remains a medical emergency associated with significant morbidity and mortality, especially in the older population. The failure rate of treatment is high, especially with delay in diagnosis and treatment. Furthermore, neurological complications including altered mental status, cerebral edema, increased intracranial pressure, hydrocephalus, seizure, sensorineural hearing loss, focal neurological deficit, cerebrovascular abnormalities and intellectual impairment have been reported in approximately 30\% patients with BM (3). Therefore, it is important for emergency physicians to promptly diagnose this condition and institute timely management for patients. However, this is difficult as patients often present with non-specific signs and symptoms, with the classical triad of fever, headache and nuchal rigidity present in only $44 \%$ of patients (4).

Medical management is the mainstay of treatment for BM. The patient should be resuscitated and have airway, breathing and circulation stabilized. Blood and CSF samples should be obtained as soon as possible, followed by the administration of empirical antimicrobial therapy (5) (Figure 1). In addition, acyclovir may be added to the regimen when herpes simplex virus or varicella zoster virus is suspected as the causative organism, and dexamethasone may be added to known or suspected pneumococcal meningitis to reduce the incidence of hearing loss and other neurological complications. Computed tomography (CT) of the brain should be considered before lumbar puncture if the patient has any 
of these: severely impaired consciousness, focal neurological deficit, seizure, signs of increased intracranial pressure such as papilledema, or if the patient is immunocompromised (5).

However, some patients may require neurosurgical interventions that are uncommon in the management of BM. Therefore, we present a 42-year-old Chinese gentleman who presented to the ED and was subsequently diagnosed with pneumococcal meningitis. We aimed to highlight the need for emergency physicians to be cognizant of the indications for neurosurgical referral. This will ensure that timely referrals can be made to minimize delay and prevent adverse outcomes for these patients.

\section{Case Report}

A 42-year-old Chinese gentleman with no past medical history was admitted to ED by ambulance. He was found in a confused state on the floor next to his bed by his wife. He had fever with runny nose, sore throat and non-vertiginous giddiness of one day duration. He had no cough, headache, neck pain, photophobia, nausea or vomiting.

He was febrile with a body temperature of $39.1{ }^{\circ} \mathrm{C}$. His heart rate was 87 beats per minute with a blood pressure of 124/59 $\mathrm{mmHg}$. His respiratory rate was 28 breaths per minute with an oxygen saturation of $99 \%$ in room air. He was agitated with a glasgow coma scale (GCS) of 11 (E4V2M5). His pupils were equal at $3 \mathrm{~mm}$ and reactive to light. The neck was supple with negative Brudzinski's and Kernig's signs. There was no focal neurological deficit. No rash and external injuries were noted. The rest of the examination was unremarkable.

Bedside blood glucose level was $12.4 \mathrm{mmol} / \mathrm{L}$. Complete blood count result was as follows: hemoglobin=14.1 g/dL, white blood cell count $=28.5 \times 10^{3} / \mathrm{uL}(92.6 \%$ neutrophils) and platelet count $=186 \times 10^{3} / \mathrm{uL}$. C-reactive protein was $176.6 \mathrm{mg} / \mathrm{L}$, procalcitonin was $8.86 \mathrm{ng} / \mathrm{L}$ and lactate was $11.2 \mathrm{mmol} / \mathrm{L}$. Other blood examinations were unremarkable. CT of the brain revealed global cerebral edema with diffuse sulcal and basal cisternal effacement. There was also a subtle hypodensity with loss of gray-white matter differentiation in the right high parietal lobe due to focal edema. Tonsils were at the level of foramen magnum (Figures 2, 3). Chest x-ray and urine culture were unremarkable.

He received empirical treatment with intravenous ceftriaxone, vancomycin, acyclovir and dexamethasone. During his stay in ED, his GCS fell to 9 (E3V2M4) with anisocoria - $6 \mathrm{~mm}$ left pupil and $2 \mathrm{~mm}$ right pupil. He was intubated due to decline in GCS and impending brain herniation due to increased intracranial pressure. Hypertonic saline was started and urgent neurosurgery consultation was performed. He was taken to the emergency operation theatre for external ventricular drainage. Intraoperatively, the CSF pressure was high and its appearance was cloudy. Analysis of the CSF showed 996 white blood cells per $\mathrm{mm}^{3}$ with neutrophil predominance at $70 \%$, and glucose level of $4.0 \mathrm{mmol} / \mathrm{L}$ and protein level of $2.23 \mathrm{~g} / \mathrm{L}$. Gram staining revealed

\begin{tabular}{|l|l|l|}
\hline Patient Profile & Common Pathogen & Empirical Antibiotics \\
\hline Patients who are 50 years old or & Neiserria meningitidis, & Ceftriaxone or cefotaxime \\
less AND immunocompetent & Streptococcus pneumoniae & AND \\
years old & Vancomycin \\
\hline Patients who are more than 50 & Streptococcus & Ceftriaxone or cefotaxime \\
& meningitidis, Listeria & Vancomycin \\
\hline Patients who are & monocytogenes & AND \\
immunocompromised & Streptococcus & Ampicillin \\
\hline pneumoniae, Neiserria & AND \\
& meningitidis, Listeria & Vancomycin \\
& monocytogenes, & AND \\
& Pseudomonas aeruginosa & Ampicillin \\
\hline & & Aoxifloxacin \\
& & Aancomycin \\
\hline
\end{tabular}

Figure 1. Empirical antibiotics for community-acquired meningitis Note: Empirical antibiotics may vary according to institutional practice

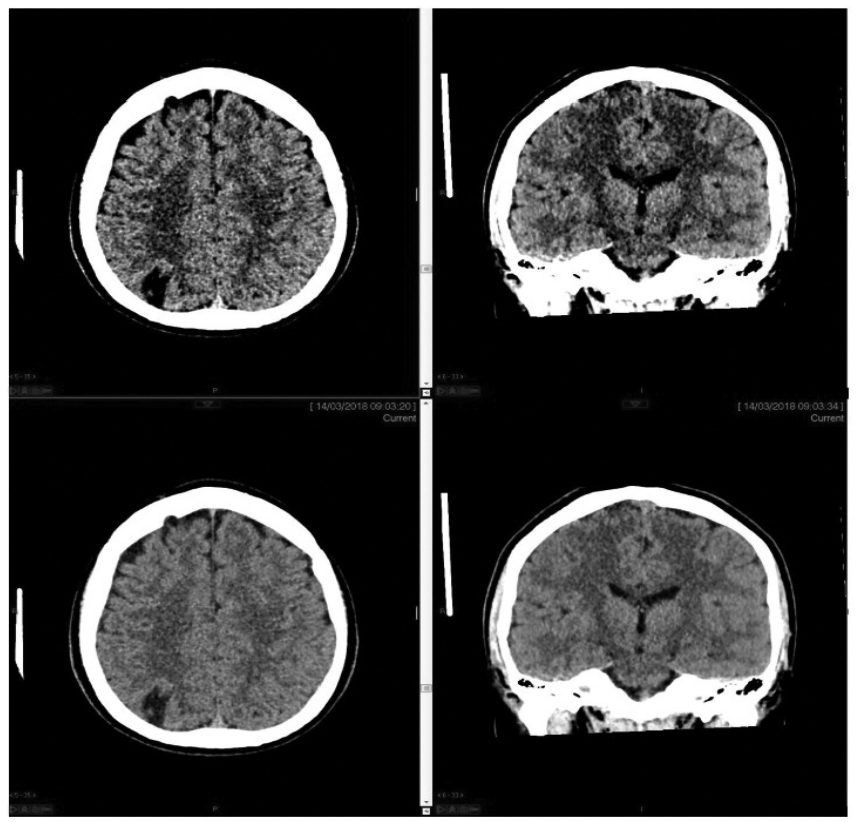

Figure 2. Brain computed tomography demonstrating loss of gray-white matter differentiation in the right high parietal lobe due to focal edema 
gram-positive cocci with Streptococcus pneumoniae detected in the PCR assay. CSF and blood cultures were positive for pansensitive Streptococcus pneumoniae.

He was admitted to the intensive care unit. During his hospitalization, he developed a new onset left sided weakness due to stroke as a vascular complication of meningoencephalitis. Magnetic resonance imaging of the brain showed acute infarcts in the right parietal and occipital lobes as well as the right centrum semi-ovale (Figure 4). He had subsequent seizure and levetiracetam was started for seizure control. Bilateral hearing loss was detected. He underwent extensive inpatient rehabilitation for his deconditioning and stroke. He was eventually discharged on day 30 with independent function and performance in Activities of Daily Living.

Informed consent was obtained from the patient for this case report.
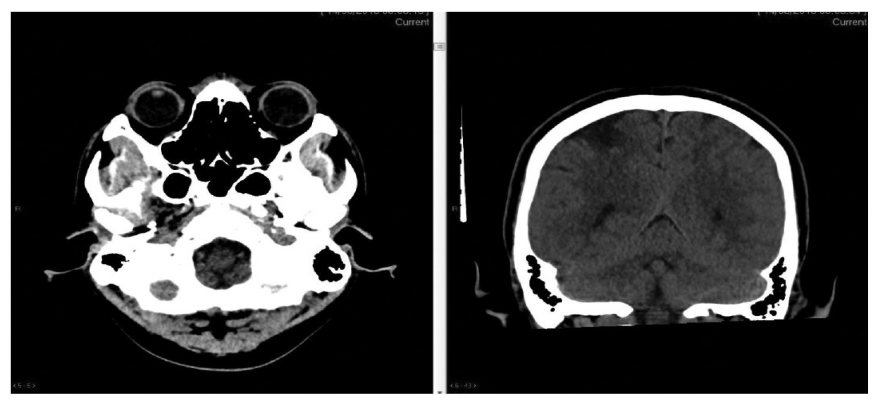

Figure 3. Brain computed tomography demonstrating global cerebral edema with diffuse sulcal and basal cisternal effacement and tonsils are seen at the level of foramen magnum

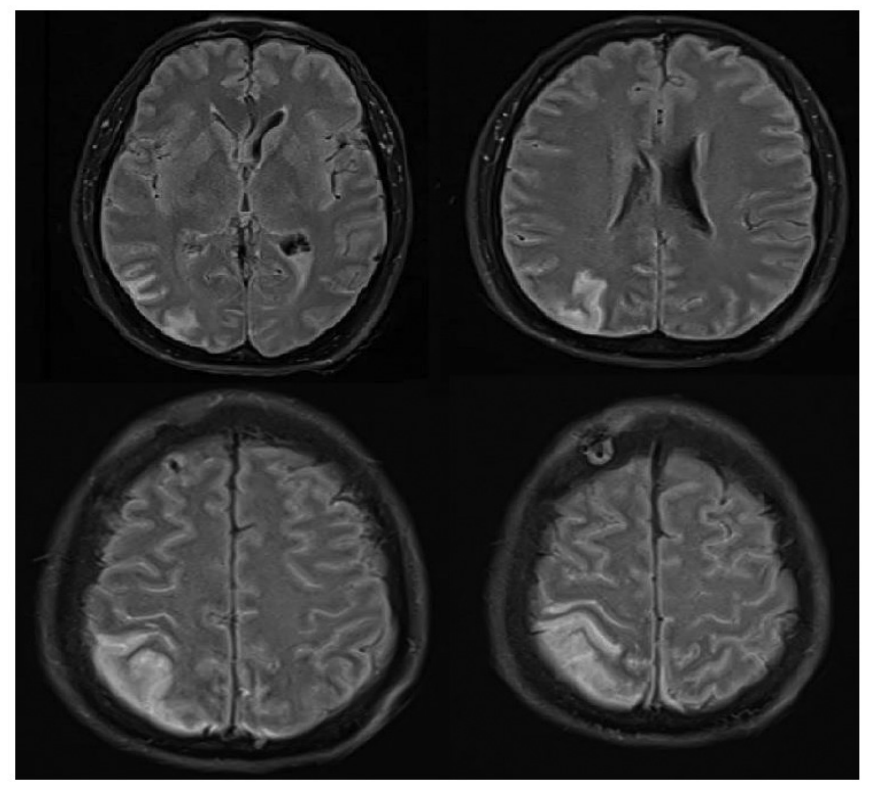

Figure 4. Brain magnetic resonance imaging demonstrating acute infarcts in the right parietal and occipital lobes

\section{Discussion}

A neurosurgery consultation should be performed if meningitis is associated with a surgically treatable lesion identified in brain $\mathrm{CT}$, development of neurological complications such as increased intracranial pressure or hydrocephalus, or inadequate treatment response to appropriate antibiotic therapy (6). Among these, the first two indications are more relevant to the setting of the ED.

Our patient was referred to neurosurgery department due to concerns of increased intracranial pressure with impending brain herniation. This was attributable to the global cerebral edema without any space-occupying lesion in brain CT. Cerebral edema occurs in BM as a result of three mechanisms: vasogenic edema from increased permeability of the blood-brain barrier, cytotoxic edema from release of cytokines from neutrophils and bacteria, and interstitial edema from abnormal absorption of cerebrospinal fluid from the subarachnoid space (5). While dexamethasone is associated with worse outcome in other conditions of increased intracranial pressure such as in head injury and cerebral infarction, dexamethasone given for BM can reduce intracranial pressure and increase vascular perfusion. Therefore, in addition to other standard therapies such as head elevation, sedation and hypertonic saline or mannitol administration, treatment for increased intracranial pressure may be given based on suspicion on clinical ground prior to imaging.

Other neurological complications that may require neurosurgery consultation in ED include cerebrovascular abnormalities such as intracranial hemorrhage, hydrocephalus, mycotic aneurysm and brain abscess. The emergency physician must actively assess for these complications, so that timely intervention by neurosurgeons can improve patient outcomes.

The incidence of neurological complications is higher in patients with meningitis due to Streptococcus pneumonia than other organisms (3). This was the case in our patient who developed left hemiparesis, seizure and bilateral hearing loss. In BM, stroke is most commonly due to arterial infarction and occurs in about one third of the patients. Among these, more than half have blood vessel abnormalities such as wall irregularities, arterial occlusion, as well as focal dilatations and bleeding, suggestive of vasculitis (7). Therefore, dexamethasone should be used instead of standard treatment with thrombolytics. Fortunately, most patients recover with resolution of their neurological deficits following successful treatment.

Similarly, about one third of patients with BM develop seizures due to the presence of bacterial toxins and the neurochemical changes following inflammation, both lowering the seizure threshold (8). In addition to the use of routine anti-epileptics, 
dexamethasone should also be administered to reduce the inflammatory response. These patients may develop recurrent seizures and therefore, anti-epileptics should be continued until a seizure-free period of three months.

Approximately $20 \%$ of patients with BM develop sensorineural hearing loss as a result of direct bacterial invasion and inflammatory response causing damage to the eighth cranial nerve, cochlea or labyrinth (9). Once again, dexamethasone has a therapeutic role in these cases.

Although dexamethasone appears to be a useful drug for the prevention and treatment of neurological complications in BM, it should be noted that its efficacy varies in different patient populations. In developing countries, dexamethasone did not show significant benefit in BM due to poor nutrition, delayed presentation, presence of chronic diseases such as infection with Human Immunodeficiency Virus and inadvertent inclusion of cases of tuberculous meningitis (10). Therefore, the practice of administrating dexamethasone with antibiotics may be limited to the developed countries.

\section{Conclusion}

$\mathrm{BM}$ is a medical emergency that requires early diagnosis and timely management. While the mainstay of management is medical, selected patients may require neurosurgical intervention. The emergency physician should be aware of these rare but important indications so that patients may benefit from early referrals and have better clinical outcomes. Although the etiology of meningitis may not be obvious in ED, dexamethasone should be administered early with antibiotics for suspected cases of meningitis in developed countries given its important role in the prevention and treatment of neurological complications.

\section{Ethics}

Ethics Committee Approval: This case report did not require approval as per Institutional Review Board guidelines.

Informed Consent: The patient gave informed consent for this case report for publication.
Peer-review: Externally and internally peer-reviewed.

\section{Authorship Contributions}

Surgical and Medical Practices: J.H.P., Concept: J.H.P., Design: J.H.P., Literature Search: M.J.L., J.H.P., Writing: M.J.L., J.H.P.

Conflict of Interest: No conflict of interest was declared by the authors.

Financial Disclosure: The authors declared that this study received no financial support.

\section{References}

1. Kottapally M, Josephson SA. Common neurologic emergencies for nonneurologists: when minutes count. Cleve Clin J Med. 2016;83:116-26.

2. Fitch MT, van de Beek D. Emergency diagnosis and treatment of adult meningitis. Lancet Infect Dis. 2007;7:191-200.

3. Durand ML, Calderwood SB, Weber DJ, Miller SI, Southwick FS, Caviness VS Jr et al. Acute bacterial meningitis in adults. A review of 493 episodes. N Engl J Med. 1993;328:21-8

4. van de Beek D, de Gans J, Spanjaard L, Weisfelt M, Reitsma JB, Vermeulen M. Clinical features and prognostic factors in adults with bacterial meningitis. $\mathrm{N}$ Engl J Med. 2004;351:1849-59.

5. Tunkel AR, Hartman BJ, Kaplan SL, Kaufman BA, Roos KL, Scheld WM, et al Practice guidelines for the management of bacterial meningitis. Clin Infect Dis. 2004;39:1267-84

6. Namani SA, Koci RA, Kuchar E, Dedushi KH. Surgical treatment of neurologic complications of bacterial meningitis in children in Kosovo. J Trop Pediatr. 2012;58:139-42

7. Pfister HW, Borasio GD, Dirnagl U, Bauer M, Einhäupl KM. Cerebrovascular complications of bacterial meningitis in adults. Neurology. 1992;42:1497504.

8. Feldman WE. Relation of concentrations of bacteria and bacterial antigen in cerebrospinal fluid to prognosis in patients with bacterial meningitis. N Engl J Med. 1977;296:433-5.

9. Kastenbauer S, Pfister HW. Pneumococcal meningitis in adults: spectrum of complications and prognostic factors in a series of 87 cases. Brain 2003;126:1015-25

10. Scarborough M, Gordon SB, Whitty CJ, French N, Njalale Y, Chitani A, et al. Corticosteroids for bacterial meningitis in adults in sub-Saharan Africa. $\mathrm{N}$ Engl J Med. 2007;357:2441-50. 